\title{
Phacoemulsification with Posterior Sub-Tenon Triamcinolone Acetonide Injection for Prevention of Progression of Diabetic Macular Edema after Cataract Surgery
}

\author{
Hassan Mohamed Hegazy, Mahmoud Mohammed Ahmed Ali, Mohamed Abd elmoneim \\ Mohamed Sedik Ghoneimy*
}

Department of Ophthalmology, Faculty of Medicine, Al-Azhar University

*Corresponding author: Mohamed Abd elmoneim Mohamed Sedik Ghoneimy, Mobile: +201288999633,

E-Mail: dr.mohamedsedik@live.co.uk

\begin{abstract} intraocular pressure (IOP) were recorded. compared to values at one month. cataract and DME.

Introduction

Diabetic maculopathy is the most common cause of visual impairment in diabetic retinopathy (DR) and is manifested most frequently as diabetic macular edema (DME) (1).
\end{abstract}

Background: diabetic macular edema (DME) is the most common cause of visual loss in diabetic patients. The pathogenesis of DME is multifactorial. It results from multiple biochemical and cellular changes that eventually cause leakage and exudation. A number of patient characteristics such as increasing age, females, duration of DM, poor glycemic control and moderate to severe retinopathy have been found associated with poor prognosis after cataract surgery in diabetic patients.

Objective: the aim of this study was to evaluate the efficacy of phacoemulsification with posterior sub-Tenon's triamcinolone acetonide injection for prevention of progression of diabetic macular edema following uneventful cataract surgery by phacoemulsification.

Patients and Methodology: this interventional study included 20 eyes of 20 diabetic patients with visually significant cataract and coexisting DME during the period from December 2017 to October 2018. Preoperative and postoperative best corrected visual acuity (BCVA) using LogMAR and

Results: statistically significant improvement in BCVA was detected when comparing postoperative values at one week, one month and three months to corresponding preoperative values. In addition, values at one month and three months were significantly improved when compared to values at one week post-operative. On other hand, values at three months revealed non-significant difference when

Conclusion: this study suggests that posterior sub-Tenon's triamcinolone acetonide injection can be given safely and easily at the time of phacoemulsification surgery in patients with visually significant

Keywords: Sub-Tenon's, Triamcinolone acetonide, Diabetic retinopathy, DME, OCT

The three primary tools used by ophthalmologists in the evaluation of DME are slit-lamp biomicroscopy, fluorescein fundus angiography (FFA), and macular optical coherence tomography (OCT) ${ }^{(2)}$.

FFA has been in use for over half a century and can identify vascular abnormalities including microaneurysms, neovascularization (NV), intra-retinal microvascular abnormalities (IRMA), capillary non-perfusion and leakage. However, leakage detected by FFA does not necessarily correspond to macular edema (ME) ${ }^{(3)}$.

Only OCT can measure central macular thickness (CMT) objectively. This is important because no observation correlates better with visual acuity (VA) in DME than axial retinal thickening, although the degree to which retinal thickening corresponds to VA depends on the pattern of DME seen ${ }^{(4)}$.

Additionally, OCT is the best modality for visualizing and evaluating pathology of the vitreomacular interface, including epiretinal membranes (ERM) and vitreomacular traction (VMT), which may contribute to the persistence of DME after focal laser photocoagulation or intravitreal pharmacotherapy with anti-vascular endothelial growth factor (VEGF), and it can also identify patients who may benefit from pars plana vitrectomy (PPV) and membrane peeling (MP) ${ }^{(5)}$.

It has been demonstrated that DR progresses in approximately $10-30 \%$ of patients after cataract surgery, although some authors have hypothesized that the progression of DR after cataract surgery is due simply to the natural 
course of the condition, and that the progression is independent of the surgery ${ }^{(6)}$.

Distinguishing transient $\mathrm{ME}$ from substantial progression of maculopathy is important to the timing of treatment for the DME. Laser photocoagulation, vitrectomy, intravitreal injections of anti-VEGF and triamcinolone acetonide (TA) are different available options for DME. However, until recently, there had been no quantitative study to examine the progression of DME after cataract surgery ${ }^{(7)}$.

Intravitreal TA (IVTA) injection is a promising treatment for DME but linked to elevation of intraocular pressure (IOP), retinal detachment (RD), glaucoma, intraocular hemorrhage and endophthalmitis ${ }^{(8)}$.

\section{Aim of Work:}

To evaluate the efficacy of phacoemulsification with posterior subTenon's triamcinolone acetonide injection for prevention of progression of diabetic macular edema following uneventful cataract surgery by phacoemulsification.

Patients and Methods

\section{A. Patients:}

\section{Patient Selection:}

This is $\begin{array}{r}\text { an } \\ \text { study }\end{array}$
interventional of
comprised of 20 eyes of
diabetic patients with cataract
and coexisting CSME going
for phacoemulsification
cataract surgery with posterior
sub-Tenon's triamcinolone
acetonide ryjection.
Preoperative and postoperative
BCVA, CMT and IOP were
recorded. The study was
approved by the Ethics
Board of Al-Azhar
University.

B. Methods:

Evaluation at the entry of this study (Preoperative evaluation) by:

\section{History taking:}

1. Onset, course and duration of diminution of vision.

2. History of ocular trauma, ocular surgery, systemic disorder and drug intake.

\section{General examination:}

Review for systemic diseases as hypertension, bleeding tendency and renal impairment.

\section{Laboratory investigation:}

Fasting blood glucose, 2 hours postprandial blood glucose, HbA1c, Coagulation profile, Liver and Kidney function tests.

\section{Preoperative \\ ophthalmological examination:}

1. Unaided visual acuity (UAVA) and BCVA using LogMAR.

2. Slit-lamp biomicroscopy examination to assess corneal clarity, depth of anterior chamber, lens morphology, state of pupil dilatation and reaction.

3. IOP measurement by Goldman Applanation tonometer.

4. Fundus examination: slit-lamp biomicroscopy using noncontact Volk 90 Diopter lens.

5. Assessment of ocular motility in all directions of gaze.

6. Examination of ocular adnexa.

\section{Preoperative} ophthalmological investigation:

1. Calculation of IOL power and axial length by Sonomed Biometry model 5500.

2. FFA by Zeiss Visucam fundus camera. (Fig. 1).

3. OCT by Topcon 3D 2000 OCT (Fig. 2). 


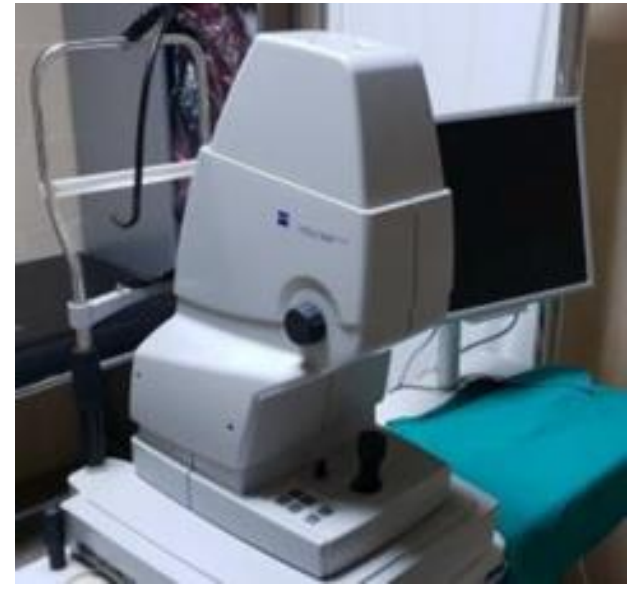

Figure (1): Zeiss VISUCAM fundus camera

\section{OCT scanning:}

- Mydriatic eye drops was instilled into patients eyes prior to OCT examination to achieve as much pupil dilatation as we can to assure maximal OCT signal and analysis.

- The patient's chin was positioned in the chin rest.

- The patient is then asked to fixate on a target inside the instrument.

- The alignment phase is completed by means a camera, located inside the instrument that displays the fundus and scan beam.

- After scanning of the patients was finished, analysis protocol was used to obtain circular maps centered on the fovea. It includes also numerical values for the foveal thickness in microns and other values measuring macular thickness divided into quadrants centered on the foveal center.

- Measurement results are presented using assigned colors to indicate retinal thickness in the region under analysis (Fig. 3\&4).

1- Green: the thickness within normal according sex and age.

2- Yellow: moderate thinning.

3- Red: severe thinning.

4- Orange: moderate thickening.

5- Purple: severe thickening.

The macular retinal map as seen in figure, divides the region into a central disc with a radius of 500 microns, and two concentric rings divided into four quadrants. The analysis program reports the corresponding mean thickness in each of the areas. The scan

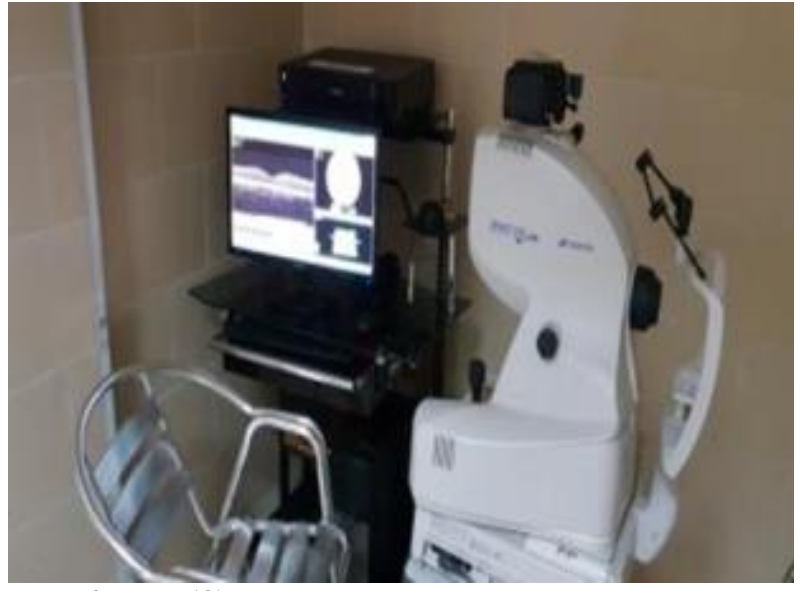

Figure (2): Topcon 3D 2000 OCT.

of a normal retina in the macular region appears green (200 to 250 microns).

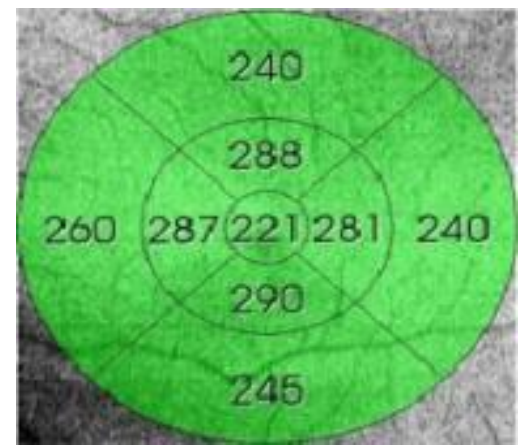

Figure (3): Normal macula and distribution of its thickness in the form of a coloured map.

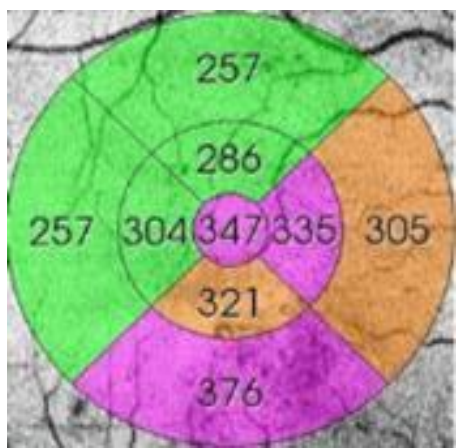

Figure (4): Abnormal macula and distribution of its thickness in the form of a coloured map. 
* Procedure:

- All pupils were dilated with $1 \%$ tropicamide and $2.5 \%$ phenylephrine.

- Ocular sterilization was done using Povidine iodine $5 \%$ application to the ocular surface for full asepsis.

- Retrobulbar or peribulbar local anesthesia.

- Clear corneal incision using keratome (2.4 or $2.8 \mathrm{~mm}$ ) was done.

- $\quad$ Two side ports were made by MVR 20 gauge or Superblade.

- Formation of the anterior chamber by viscoelastic material, continuous circular curvilinear capsulorhexis performed under viscoelastic material started with bent insulin needle and completed with capsulorhexis forceps followed by hydro dissection and hydro delineation.

- Phacoemulsification was done by Constellation machine (Alcon, Forte Worth, TX, USA) using the following steps:

1. Stop and chop technique was the main technique used for phacoemulsification.

2. started by phaco one to make a groove (vacuum from 100-150, torsional power $60-80$, longitudinal power 10-30) the direction is from 11 o'clock to 7 o'clock, the groove is deepened till there is a faint part of the nucleus then divide the nucleus into 2 parts.

3. Phaco two was done to each part, using chopper to fragment the nucleus into smaller pieces which makes emulsification as well as the aspiration of cortical material easier by pulsed mode (vacuum 250-300 and power torsional 50-60 and longitudinal 10-20).

4. Automated bimanual irrigation aspiration of cortical matter after removal of all nuclear quadrants was used.

5. The capsular bag was inflated with viscoelastic material, after which the acrylic intraocular lens injected in the bag, and then removal of viscoelastic by automated I/A cannulas.

6. Finally, hydration of the wound and the two paracentesis ports. 
- At the completion of cataract surgery all patients were injected by triamcinolone acetonoide via incision of the bulbur conjunctiva $8 \mathrm{~mm}$ from the limbus at the infero-temporal quadrant, then the Tenon's capsule is dissected through the wound inferior and posterior to the globe, then $1 \mathrm{ml}$ of $40 \mathrm{mg} / \mathrm{ml}$ of triamcinolone acetonide is injected using viscoelastic gauge or blunt 19 gauge infusion cannula and insulin syringe followed by massaging and compression on the conjunctival wound, (Fig. 5).

- Eyes that had any intraoperative or postoperative complications were excluded from the study.

- After the operation all patients were received the same standard medications for four weeks, consisting of a combination of steroid (Prednisolone acetate 1\%) and antibiotic (Moxifloxacin 0.5\%) eye drops beginning with five times daily, and tapered gradually.

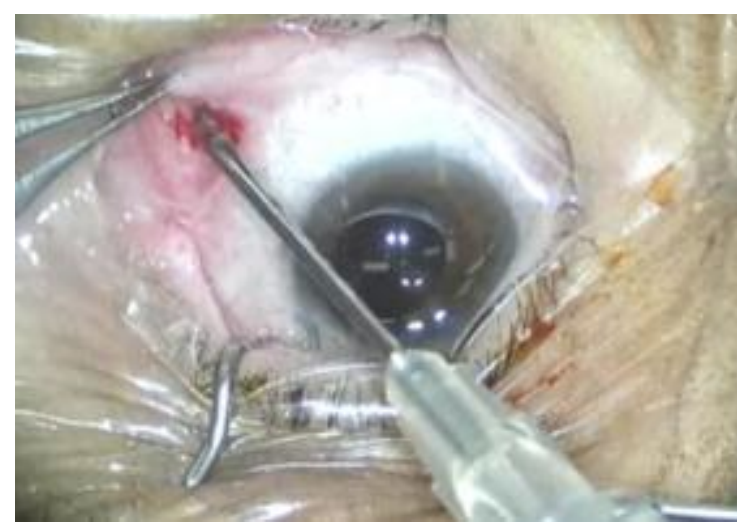

Figure (5): Sub-Tenon's TA injection after the completion of phacoemulsification surgery.

\section{* Post operative follow up:}

The assessment was done one day post operative followed by one week, one month and three months post operative follow up.

\section{One day after surgery:}

1. Slit lamp examination for:

a) Exclusion of any intraocular or extra-ocular reaction or infection.

b) State of main incision and exclusion of any leakage or iris prolapse.

c) Corneal examination for clarity, edema and ulcers.

d) Anterior chamber (depth and contents).

e) Any iris abnormality.

f) Intraocular lens regarding its position and any deposits on its surface.

2. Intraocular pressure (IOP).

One Week after surgery:

1- BCVA (LogMAR).

2- Slit-lamp examination.

3- Intraocular pressure (IOP).

4- Fundus examination by slit-lamp biomicroscopy using non-contact Volk 90 Diopter lens.

\section{One month after surgery:}

1- BCVA (LogMAR).

2- Slit-lamp examination.

3- Intraocular pressure (IOP).

4- Fundus examination by slit lamp biomicroscopy using non-contact Volk 90 Diopter lens.

5- Optical coherence tomography (OCT).

Three months after surgery:

1- BCVA (LogMAR).

2- Slit-lamp examination.

3- Intraocular pressure (IOP).

4- Fundus examination by slit lamp biomicroscopy using non-contact Volk 90 Diopter lens.

5- Optical coherence tomography (OCT).

6- Fluorescein fundus angiography (FFA).

\section{Statistical analysis:}

Data were analyzed using Statistical Program for Social Science (SPSS) version 18.0. Quantitative data were expressed as mean \pm standard deviation (SD). Qualitative data were expressed as frequency and percentage.

- $\quad$ Probability (P-value)

- $\quad$ P-value $<0.05$ was considered significant.(Sig)

- $\quad$ P-value 0.01 was considered as highly significant.(HS)

- $\quad$ P-value >0.05 was considered non-significant.(NS)

Informed consent and approval:

All patients had informed written consent and approval, revised their history, investigations and explained the procedure to them. 
Phacoemulsification with Posterior Sub-Tenon Triamcinolone Acetonide...

\section{Results}

Table (1): Distribution of studied patients regarding their age

\begin{tabular}{|l|c|}
\hline & Age \\
\hline Minimum & 45.00 \\
\hline Maximum & 68.00 \\
\hline Mean & 57.80 \\
\hline S D & 6.67 \\
\hline
\end{tabular}

Age of patients in the present work ranged from 45 to 68 years; the mean age was 57.80 years and the standard deviation was 6.67 years.

Table (2): Distribution of studied patients regarding their gender

\begin{tabular}{|c|c|c|c|}
\hline & & Number & $\%$ \\
\hline \multirow{3}{*}{ Sex } & Male & 5 & 25.0 \\
\hline & Female & 15 & 75.0 \\
\hline & Female : Male ratio & \multicolumn{2}{|c|}{$3: 1$} \\
\hline
\end{tabular}

In the present work, there were 5 males $(25.0 \%)$ and 15 females (75.0\%) with male to female ratio of $3: 1$.

Table (3): Preoperative measurements

\begin{tabular}{|c|c|c|c|c|}
\hline & Minimum & Maximum & Mean & SD \\
\hline BCVA $^{1}$ & 1.3 & 0.7 & 1.03 & 0.215 \\
\hline CMT $^{\mathbf{1}}$ & $321.00 \mu \mathrm{m}$ & $805.00 \mu \mathrm{m}$ & $489.04 \mu \mathrm{m}$ & 120.51 \\
\hline IOP $^{2}$ & $11.00 \mathrm{mmHg}$ & $17.00 \mathrm{mmHg}$ & $14.00 \mathrm{mmHg}$ & 1.44 \\
\end{tabular}

${ }^{1}$ Best corrected visual acuity, ${ }^{2}$ Central macular thickness, ${ }^{3}$ Intra ocular pressure

Regarding preoperative measurements, BCVA ranged from 1.3 to 0.7 with the mean $\pm \mathrm{SD}$ of $1.03( \pm 0.215)$; the CMT ranged from $321.0 \mu \mathrm{m}$ to $805.0 \mu \mathrm{m}$ with mean \pm SD of $489.04( \pm 120.51)$; the preoperative IOP ranged from 11.0 to $17.0 \mathrm{mmHg}$ with the mean $\pm \mathrm{SD}$ of $14.0( \pm 1.44) \mathrm{mmHg}$.

Table (4): Postoperative measurements in studied populations

\begin{tabular}{|c|c|c|c|c|c|}
\hline \multicolumn{2}{|c|}{ Postoperative } & Mean & SD & Minimum & Maximum \\
\hline One day & IOP & $18.0 \mathrm{mmHg}$ & 1.44 & $16.00 \mathrm{mmHg}$ & $21.00 \mathrm{mmHg}$ \\
\hline \multirow{3}{*}{ One week } & BCVA & 0.58 & 0.101 & 0.7 & 0.5 \\
\cline { 2 - 6 } & IOP & $15.96 \mathrm{mmHg}$ & 4.51 & $12.00 \mathrm{mmHg}$ & $28.00 \mathrm{mmHg}$ \\
\hline \multirow{3}{*}{ One month } & BCVA & 0.45 & 0.248 & 1.0 & 0.2 \\
\cline { 2 - 6 } & CMT & $239.48 \mu \mathrm{m}$ & 48.88 & $119.00 \mu \mathrm{m}$ & $331.00 \mu \mathrm{m}$ \\
\cline { 2 - 6 } & IOP & $12.92 \mathrm{mmHg}$ & 1.15 & $11.00 \mathrm{mmHg}$ & $15.00 \mathrm{mmHg}$ \\
\hline \multirow{3}{*}{ Three months } & BCVA & 0.41 & 0.199 & 1.0 & 0.2 \\
\cline { 2 - 6 } & CMT & $243.68 \mu \mathrm{m}$ & 62.78 & $162.00 \mu \mathrm{m}$ & $431.00 \mu \mathrm{m}$ \\
\cline { 2 - 6 } & IOP & $12.36 \mathrm{mmHg}$ & 0.95 & $11.00 \mathrm{mmHg}$ & $14.00 \mathrm{mmHg}$ \\
\hline
\end{tabular}

At the first postoperative day, IOP ranged from $16.0 \mathrm{mmHg}$ to $21.0 \mathrm{mmHg}$ with a mean of 18.0 $( \pm 1.44) \mathrm{mmHg}$. At the first week, BCVA ranged from 0.7 to 0.5 with a mean of $0.58( \pm 0.101)$, while IOP ranged from $12 \mathrm{mmHg}$ to $28 \mathrm{mmHg}$ with mean of $15.96( \pm 4.51) \mathrm{mmHg}$. At $1^{\text {st }}$ month postoperative, the BCVA ranged from 1.0 to 0.2 with a mean of $0.45( \pm 0.248)$, while CMT ranged from $119 \mu \mathrm{m}$ to $331.0 \mu \mathrm{m}$ with a mean of $239.48( \pm 48.88) \mu \mathrm{m}$, and IOP ranged from $11 \mathrm{mmHg}$ to $15 \mathrm{mmHg}$ with a mean of $12.92( \pm 1.15) \mathrm{mmHg}$. Finally, at $3^{\text {rd }}$ month post operative, the BCVA ranged from 1.0 to 0.2 with a mean of $0.41( \pm 0.199)$, while CMT ranged from $162 \mu \mathrm{m}$ to $431 \mu \mathrm{m}$ with a mean of 243.68 $( \pm 62.78) \mu \mathrm{m}$ and IOP ranged from $11 \mathrm{mmHg}$ to $14 \mathrm{mmHg}$ with a mean of $12.36( \pm 0.95) \mathrm{mmHg}$ respectively.

Table (5): Repeated measures ANOVAs for BCVA

\begin{tabular}{|l|c|c|c|c|}
\hline & Mean & S. D & F & P \\
\hline Preoperative & 1.03 & 0.215 & & \\
\hline
\end{tabular}




\begin{tabular}{|c|c|c|c|c|}
\hline One week $\mathrm{PO}^{3}$ & 0.58 & 0.101 & \multirow{3}{*}{161.36} & \multirow{3}{*}{$<0.001 *$} \\
\hline One month PO & 0.45 & 0.248 & & \\
\hline Three months PO & 0.41 & 0.199 & & \\
\hline \multicolumn{3}{|c|}{ Preoperative Vs One week postoperative } & Diff $=0.45$ & $<0.001 *$ \\
\hline \multicolumn{3}{|c|}{ Preoperative Vs One month postoperative } & Diff $=0.58$ & $<0.001 *$ \\
\hline \multicolumn{3}{|c|}{ Preoperative Vs Three months postoperative } & Diff $=0.62$ & $<0.001 *$ \\
\hline \multicolumn{3}{|c|}{ One week Vs One month postoperative } & Diff $=0.13$ & $<0.001 *$ \\
\hline \multicolumn{3}{|c|}{ One week Vs Three months postoperative } & Diff $=0.17$ & $<0.001 *$ \\
\hline \multicolumn{3}{|c|}{ One month Vs Three months postoperative } & Diff $=0.04$ & $0.072(\mathrm{~ns})$ \\
\hline
\end{tabular}

When comparing postoperative values at one week, one month and three months to corresponding preoperative value, there was statistically significant increase of numerical values of BCVA at all postoperative measurements when compared to preoperative values. In addition, values at one week, one month and three months were significantly increased when compared to values at the first postoperative day. Also, values at one month and three months were significantly increased when compared to values at one week postoperative. On other hand, values at three months revealed nonsignificant difference when compared to values at one month.

Table (6): Repeated measures ANOVAs for CMT

\begin{tabular}{|l|c|c|c|c|}
\hline & Mean & SD & F & p \\
\hline Preoperative & $489.04 \mu \mathrm{m}$ & 120.51 & \multirow{2}{*}{95.18} & \\
\hline One month PO & $239.48 \mu \mathrm{m}$ & 48.88 & & \\
\hline Three months PO & $243.68 \mu \mathrm{m}$ & 62.78 & & $<0.001^{*}$ \\
\hline Preoperative vs. One month postoperative & & Diff $=249.56$ & $<0.001^{*}$ \\
\hline Preoperative vs. three months postoperative & Diff $=245.36$ & $0.74(\mathrm{~ns})$ \\
\hline
\end{tabular}

When comparing postoperative values of CMT with preoperative values, the values at one month and three months postoperative, were significantly decreased when compared to preoperative values. On the other hand, values at three months showed non-significant difference when compared to values at one month postoperative.

Table (7): Repeated measures ANOVAs for IOP

\begin{tabular}{|c|c|c|c|c|}
\hline & Mean & S. D & $\mathbf{F}$ & $\mathbf{P}$ \\
\hline Preoperative & $14.00 \mathrm{mmHg}$ & 1.44 & \multirow{5}{*}{155.15} & \multirow{5}{*}{$<0.001 *$} \\
\hline One day PO & $18.00 \mathrm{mmHg}$ & 1.44 & & \\
\hline One week PO & $15.96 \mathrm{mmHg}$ & 4.51 & & \\
\hline One month PO & $12.92 \mathrm{mmHg}$ & 1.15 & & \\
\hline Three months & $12.36 \mathrm{mmHg}$ & 0.95 & & \\
\hline \multicolumn{3}{|c|}{ Preoperative vs. One day PO } & Diff $=4.0$ & $<0.001^{*}$ \\
\hline \multicolumn{3}{|c|}{ Preoperative vs. One week PO } & Diff $=1.96$ & $<0.001^{*}$ \\
\hline \multicolumn{3}{|c|}{ Preoperative vs. One month PO } & Diff $=1.08$ & $<0.042 *$ \\
\hline \multicolumn{3}{|c|}{ Preoperative vs. three months PO } & Diff $=1.64$ & $0.001^{*}$ \\
\hline \multicolumn{3}{|c|}{ One day vs. One week PO } & Diff $=2.04$ & $0.047 *$ \\
\hline \multicolumn{3}{|c|}{ One day vs. One month PO } & Diff $=5.08$ & $<0.001^{*}$ \\
\hline \multicolumn{3}{|c|}{ One day vs. three months PO } & Diff $=5.64$ & $<0.001^{*}$ \\
\hline \multicolumn{3}{|c|}{ One week vs. One month PO } & Diff $=3.04$ & $<0.001^{*}$ \\
\hline \multicolumn{3}{|c|}{ One week vs. three months PO } & Diff $=3.60$ & $<0.001^{*}$ \\
\hline \multicolumn{3}{|c|}{ One month vs. three months PO } & Diff $=0.56$ & $0.001^{*}$ \\
\hline
\end{tabular}

Regarding IOP, there was statistically significant increase of IOP at the first day postoperative and first week postoperative when compared to preoperative values. Then, there was significant decrease at one month and three months when compared to preoperative values. In addition, there was significant decrease of IOP at one week, one month and

\footnotetext{
${ }^{3}$ Post operative
} 
three months when compared to first postoperative day. Also, there was significant decrease at one month and three months when compared to one week postoperative. Finally, there was significant decrease at 3 months when compared to values at one month postoperative.

\section{Discussion}

DME is the most common cause of visual loss in diabetic patients ${ }^{(9)}$. A number of patient characteristics such as increasing age, females, duration of DM, poor glycemic control and moderate to severe retinopathy have been found associated with poor prognosis after cataract surgery in diabetic patients ${ }^{(\mathbf{1 0})}$.

This study conducted on 20 eyes of 20 patients during the period from December 2017 to October 2018. We assessed BCVA and CMT after the combination of uncomplicated phacoemulsification and posterior sub-Tenon's triamcinolone acetonide injection in patients with cataract and DME.

In this study all patients underwent phacoemulsification and in-the-bag intraocular lens implantation with a self-sealing corneal tunnel and posterior sub-Tenon $1 \mathrm{ml}$ $(40 \mathrm{gm} / \mathrm{ml})$ triamcinolone acetonide injection. $75 \%$ of patients were females, the mean age was 57.80 years and the mean duration of DM was 15.55 years.

Several studies have shown that phacoemulsification can result in exacerbation of ME in diabetic patients. Khedr (11) demonstrated that $20 \%$ of diabetic patient show increase in CMT after uncomplicated phacoemulsification. Liu et al. ${ }^{(\mathbf{1 2})}$ showed that uncomplicated phacoemulsification with intraocular lens implantation causes a significant increase in subclinical thickening in the region of the central macula in diabetic patients with mild to moderate NPDR at postoperative one, three and six months.

Chen et al. ${ }^{(13)}$ have shown that there is no increase in risk of progression, suggesting that the risk is less with modern phacoemulsification techniques. Short-time phacoemulsification with small self-sealed corneal incisions without iris trauma, and inthe-bag implantation of intraocular lenses, in general do not cause progression of DR.

In this study the BCVA during the three months of follow-up period after a successful phacoemulsification with $1 \mathrm{ml} \quad(40 \mathrm{mg} / \mathrm{ml})$ posterior sub-Tenon's triamcinolone acetonide injection was significantly higher than the initial BCVA.

When comparing postoperative values at one week, one month and three months to corresponding preoperative value, there was statistically significant increase of numerical values of BCVA at all postoperative measurements when compared to preoperative values. In addition, values at one week, one month and three months were significantly increased when compared to values at the first postoperative day. Also, values at one month and three months were significantly increased when compared to values at one week postoperative. On other hand, values at three months revealed non-significant difference when compared to values at one month.

In a study, Ahmadabadi et al. (14) reported that intravitreal injection of TA reduced the amount of CMT after phacoemulsification in eyes of diabetic patients. It also reduced the incidence of CME but it had no effect on VA gain.

Anatomically, in this study, the CME of patients who had phacoemulsification together with posterior sub-Tenon's triamcinolone acetonide injection showed reduction to a minimum CMT achieved at one month and was sustained at three month.

When comparing postoperative values of CMT with preoperative values (mean= $489.04 \pm 120.51) \mu \mathrm{m}$, the values at one month postoperative (mean $=239.48 \pm 48.88) \mu \mathrm{m}$ and three months postoperative $($ mean $=243.68$ $\pm 62.78) \mu \mathrm{m}$, were significantly decreased with compared to preoperative values. On the other hand, values at three months showed nonsignificant difference when compared to values at one month postoperative. Lodhi et al. ${ }^{(15)}$ demonstrated that the mean preoperative CMT on OCT was $231.16 \pm 40.86 \mu \mathrm{m}$ in the control group and $288.83 \pm 115.87 \mu \mathrm{m}$ in the TA group $(P=0.07)$. The mean CMT was $304.33 \pm$ $115.38 \mu \mathrm{m}$ and $281.50 \pm 163.74 \mu \mathrm{m}$, respectively $(P=0.63)$, postoperatively, at the end of 3 months. The mean change in CMT at 3 months was statistically significantly greater in the control group $(P=0.006)$. The CMT in the control group increased by 71 microns $(30.45 \%)$ at three months postoperatively and was statistically significant $(P=0.006)$. However, there was no statistically significant difference in the foveal thickness between the groups either at baseline $(P=0.07)$ or at three months $(P=0.63)$. At six months, there was no 
significant difference in the mean change in CMT between the 2 groups.

Kim et al. ${ }^{(7)}$ reported in their study that the mean preoperative CMT on OCT was $204.93 \pm 39.08 \mu \mathrm{m}$ in the control group and $228.24 \pm 43.34 \mu \mathrm{m}$ in the triamcinolone group $(P=0.130)$. One month postoperatively, the mean CMT was $273.93 \pm 91.00 \mu \mathrm{m}$ and 238.76 $\pm 48.20 \mu \mathrm{m}$, respectively $(P=0.469)$. The mean change in CMT at one month was statistically significantly greater in the control group $(P=$ $0.015)$, but the difference was not statistically significant at the end of six months.

In this study, 2 eyes of 20 eyes (10\%) were found to have recurrence of ME three months after phacoemulsification and posterior sub-Tenon's triamcinolone acetonide injection.

In this study there was only 1 eye (5\%) of 20 eyes developed an increased IOP of over $21 \mathrm{mmHg}$ at the 1 st week visit. None of these patients had pre-existing raised IOP or were known steroid responders. The IOP was normalized by topical anti-glaucomatous agents.

Browning et al .(16) reported that anterior peribulbar triamcinolone injections were associated with an increased risk of IOP elevation and cataract development compared to posterior sub-Tenon's triamcinolone injections.

Potential side effects of injecting TA into the posterior sub-Tenon's capsule include IOP elevation, globe perforation (rare), central retinal artery occlusion, blepharoptosis and infection. In our study, we did not encounter any complications other than elevation of IOP over $21 \mathrm{mmHg}$ in only one eye and it was controlled by topical anti-glaucomatous agents.

All the surgeries were performed in the operating room with full asepsis, lid speculum, proper draping of the patient's eyelashes and topical povidine iodine $5 \%$ preoperatively.

There are other treatment options such as direct argon laser photocoagulation applied to focally leaking micro-aneurysms and/or grid treatment applied to areas of diffuse ME results in a substantial reduction of the risk of visual loss in eyes with $\mathrm{DME}^{(\mathbf{1 7})}$.

There are also, intravitreal injections of steroids (Triamcinolone acetonide injection), anti-VEGF drugs or pars plana vitrectomy for DME. Ranibizumab and Bevacizumab are the two main anti-VEGF drugs used commonly. Although Ranibizumab has been recently approved by the United States Food and Drug
Administration for the treatment of DME, it is expensive. Bevacizumab, which costs much less than Ranibizumab, is commonly used ${ }^{(\mathbf{1 8})}$.

\section{Conclusion}

In conclusion, we found that there is statistically significant improvement in the BCVA and diabetic macular edema after phacoemulsification in comparison with other modalities of treatment of DME with cataract surgery including; intra-vitreal anti-VEGF injection and intra-vitreal steroids, sub-Tenon's triamcinolone acetonoide is more safe avoiding intraocular complications and less incidence of IOP spike, but frequent follow up of patients is important to detect recurrent and refractory cases to be managed probably and to manage IOP fluctuations.

\section{References}

1. Williams R, Airey M, Baxter $\mathrm{H}$ et al. (2004): Epidemiology of diabetic retinopathy and macular oedema: a systematic review. Eye, 18:963-983.

2. ETDRS (Early Treatment Diabetic Retinopathy Study) (1991): Design and baseline patient characteristics ETDRS report number 7. Ophthalmology. 98:741-756.

3. Bhagat $\mathrm{N}$, Grigorian RA, Tutela A et al. (2009): Diabetic macular edema: pathogenesis and treatment. Surv. Ophthalmol., 54:1-32.

4. Shimura M, Yasuda $K$, Nakazawa $T$ et al. (2011): Visual outcome after intravitreal triamcinolone acetonide depends on optical coherence topographic patterns in patients with diffuse diabetic macular edema. Retina, 31:748-754.

5. Ghazi NG, Ciralsky JB, Shah SM et al. (2007): Optical coherence tomography findings in persistent diabetic macular edema: the vitreomacular interface. Am. J. Ophthalmol., 144:747-754.

6. Tsujikawa A, Otani A, Takanashi T et al. (1997): Long-term prognosis of extracapsular cataract extraction and intraocular lens implantation in diabetic patients. Jpn. J. Ophthalmol., 41: 319323.

7. Kim SY, Yang J, Lee YC et al. (2008): Effect of a single intraoperative subTenon injection of triamcinolone 
acetonide on the progression of diabetic retinopathy and visual outcomes after cataract surgery. J. Cataract. Refract. Surg., 34:823-6.

8. Jonas JB, Akkoyun I, Kreissig I et al. (2005): Diffuse diabetic macular oedema treated by intravitreal triamcinolone acetonide: a comparative, nonrandomised study. Br. J. Ophthalmol., 89(3):321-326.

9. Burgess PI, MacCormic IJ, Harding SP et al. (2013): Epidemiology of diabetic retinopathy and maculopathy in Africa: a systematic review.Diabet Med., 30(4): 399-412.

10. Ryan L, Tien $Y$ and Charumathi $S$ (2015): Epidemiology of diabetic retinopathy, diabetic macular edema and related vision loss. Eye Vis., 2: 17-35.

11. Khedr M (2014). Evaluation of central macular thickness changes after uncomplicated phacoemulsification in diabetic patients. Journal of American Sciences, 10(10): 153-156.

12. Liu J, Jones RE, Zhao J et al. (2015): Influence of uncomplicated phacoemulsification on central macular thickness in diabetic patients: A metaanalysis. PLOS. ONE, 10(5): 126-143.

13. Chen $X$, Wen-Jun $S$, Hong-Yuan $C$ et al. (2016): Macular edema after cataract surgery in diabetic eyes evaluated by optical coherence tomography. Int. J. Ophthalmol., 9(1): 81-85.

14. Ahmadabadi HF, Mohammadi M, Beheshtnejad H et al. (2010): Effect of intravitreal triamcinolone acetonide injection on central macular thickness in diabetic patients having phacoemulsification. J. Cataract Refract Surg., 36:917-9122.

15. Lodhi SA, Shailaja $M$ and Jehan $K$ (2015): Efficacy and safety of intraoperative posterior sub-Tenon's triamcinolone injection in cataract surgery associated with diabetic retinopathy. Int. J. Sci. Stud., 3(5):82-87.

16. Browning DJ, Network DR, Glassman AR et al. (2007): Relationship between optical coherence tomography-measured central retinal thickness and visual acuity in diabetic macular edema. Ophthalmology, 114:525-536.
17. Park YG, Kim EY and Roh YJ (2014): Laser-based strategies to treat diabetic macular edema: history and new promising therapies. Journal of Ophthalmology, 2014: 9.

18. Ozkiris A (2009): Intravitreal bevacizumab (Avastin) for primary treatment of diabetic edema. Eye, 23:616-620. 